\title{
Correction to: Diversifying the xanthine scaffold for potential phosphodiesterase 9A inhibitors: synthesis and validation
}

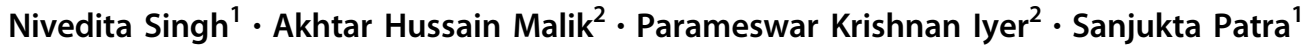

Published online: 11 June 2021

(c) Springer Science+Business Media, LLC, part of Springer Nature 2021

Correction to: Medicinal Chemistry Research

https://doi.org/10.1007/s00044-021-02722-9

published online 10 April 2021

In the published article "DIVERSIFYING THE XANTHINE SCAFFOLD FOR POTENTIAL PHOSPHODIESTERASE 9A INHIBITORS: SYNTHESIS AND VALIDATION" with Article DOI: 10.1007/s00044-02102722-9 some errors are recognized.

In the first paragraph (15th line) of the Introduction part, "Abdulrahman et al." mistakenly left in the text part, require deletion. In place of "(Ching et al. (2006); [10])" in the last line of the first paragraph of the introduction there should be "[10, 41]". Reference 41 in the original article represents this [41. He R, Ching SM, Lam Y. Traceless solid-phase synthesis of substituted xanthines. J Comb Chem. 2006;8:923-8]. In place of " $(6,11,15)$; Cai et al 2013) in the fourth line of the second paragraph of introduction, there should be " $[6,11,15,42]$ ". Reference 42 in the original article represents this. [42. Lin K, Cai Z, Wang F, Zhang W, Zhou W. Synthesis and biological evaluation of xanthine derivatives on dipeptidyl peptidase 4 . Chem Pharm Bull. 2013;61:477-82]. In place of “. ([6, 7, 11, 12, 15]; Cai et al. 2013;)" in lines 10-11 of the second paragraph of introduction, there should be " $[6,7,11,12$, $15,42] "$.

Parameswar Krishnan Iyer pki@iitg.ernet.in

$\bowtie$ Sanjukta Patra sanjukta@iitg.ernet.in

1 Nivedita Singh and Sanjukta Patra, Department of Biosciences and Bioengineering, Indian Institute of Technology Guwahati, Guwahati, Assam 781039, India

2 Akhtar Hussain Malik and Parameswar Krishnan Iyer, Department of Chemistry, Indian Institute of Technology Guwahati, Guwahati, Assam 781039, India 